\title{
Challenges from experiment: electronic structure of $\mathrm{NiO}$
}

\author{
C.-Y. Kuo ${ }^{1}$, T. Haupricht ${ }^{2}$, J. Weinen ${ }^{1,2}$, Hua Wu ${ }^{1,3}$, K.-D. Tsuei ${ }^{4}$, \\ M.W. Haverkort ${ }^{1,5}$, A. Tanaka ${ }^{6}$, and L.H. Tjeng ${ }^{1, a}$ \\ 1 Max Planck Institute for Chemical Physics of Solids, Nöthnitzer Str. 40, 01187 Dresden, \\ Germany \\ 2 Institute of Physics II, University of Cologne, Zülpicher Str. 77, 50937 Cologne, Germany \\ 3 Laboratory for Computational Physical Sciences (MOE), State Key Laboratory of \\ Surface Physics, and Department of Physics, Fudan University, Shanghai 200433, \\ P.R. China \\ 4 National Synchrotron Radiation Research Center, 101 Hsin-Ann Road, Hsinchu \\ Science-Park, Hsinchu 30076, Taiwan \\ 5 Institute for theoretical physics, Heidelberg University, Philosophenweg 19, 69120 \\ Heidelberg, Germany \\ 6 Department of Quantum Matter, ADSM, Hiroshima University, Higashi-Hiroshima \\ 739-8530, Japan
}

Received 20 February 2017 / Received in final form 24 March 2017 Published online 10 July 2017

\begin{abstract}
We report on a detailed experimental and theoretical study of the electronic structure of $\mathrm{NiO}$. The charge-transfer nature of the band gap as well as the intricate interplay between local electronic correlations and band formation makes $\mathrm{NiO}$ to be a challenging case for a quantitative ab-initio modeling of its electronic structure. To reproduce the compensated-spin character of the first ionization state and the state created by hole doping requires a reliable determination of the charge transfer energy $\Delta$ relative to the Hubbard $U$. Furthermore, the presence of non-local screening processes makes it necessary to go beyond single-site many body approaches to explain the valence band spectrum.
\end{abstract}

\section{Introduction}

$\mathrm{NiO}$ crystallizes in the rocksalt structure and is an antiferromagnetic insulator (AFI) with a Néel temperature of 523 K [1]. De Boer and Verwey [2] pointed out already 80 years ago that many properties of $3 d$ transition metal (TM) compounds cannot be explained by band theory. Indeed, standard band theory predicts $\mathrm{NiO}$ to be a metal, caused by the fact that its $3 d$ shell is partially filled. It was then understood on a qualitative level that strong Coulomb interactions between the $3 d$ electrons must play a role $[3,4]$. However, with the advancement of density functional based methods in the 1980's, it was proposed to treat the AFI oxides as Slater insulators so that a band gap can be formed [5-7]. Indeed, the calculations produced a band gap for $\mathrm{NiO}$, but its value is very small, about $0.2 \mathrm{eV}[5]$. This quantitative prediction has in fact been

\footnotetext{
${ }^{\text {a }}$ e-mail: hao.tjeng@cpfs.mpg.de
} 
falsified almost immediately by a combined photoelectron (PES) and bremsstrahlungisochromat (BIS) spectroscopy experiment which showed that the band gap is an order of magnitude larger, namely $4.3 \mathrm{eV}$ [8]. This experimental observation thus established firmly the role of strong electron correlations in determining the insulating nature of $\mathrm{NiO}$.

Nevertheless, it was not clear for a long time why the magnitude of the band gap in many correlated oxides does not always scale with the strength of the Hubbard $U$, which is defined as the energy cost to transfer one electron from one TM $3 d$ ion to another (far away) TM $3 d$ ion in the solid. This puzzle was solved by Zaanen, Sawatzky, and Alllen [9] who inferred that another energy scale plays an equally important role, namely the charge transfer energy $\Delta$, which is the energy cost to transfer an electron from an oxygen $2 p$ ion to a (far away) TM $3 d$ ion. They argue that the band gap is then determined by the smaller of the two quantities $U$ or $\Delta$. If $U$ is smaller than $\Delta$, the low energy excitations are given by the TM $3 d$ degrees of freedom so that the band gap scales with $U$. This defines a Mott-Hubbard insulator and $\mathrm{Ti}_{2} \mathrm{O}_{3}, \mathrm{~V}_{2} \mathrm{O}_{3}$, and $\mathrm{Cr}_{2} \mathrm{O}_{3}$ may serve as examples [9]. If on the other hand, $\Delta$ is smaller than $U$, then the lowest energy excitations involve the removal of an electron from the $\mathrm{O} 2 p$ and its transfer to the TM $3 d$ with the result that the band gap is given by $\Delta$. Such a system is then called a charge-transfer insulator and $\mathrm{CuO}, \mathrm{La}_{2} \mathrm{CuO}_{4}$, and $\mathrm{CuCl}_{2}$ are good representatives [9]. These findings have resulted in the conception of a new classification scheme for transition metal oxides now known as the ZaanenSawatzky-Allen (ZSA) phase diagram. Interestingly, NiO seems to have comparable $U$ and $\Delta$ values so that $\mathrm{NiO}$ can be placed in the intermediate regime of the ZSA phase diagram [9].

Taking the TM ion to have the $3 d^{n}$ configuration in the ground state, we will find for Mott-Hubbard insulators that the first ionization state or the lowest state that one can reach with hole doping, will be given by one of the $3 d^{n-1}$ multiplet states. Moreover, one can readily expect that this will be of high-spin state character since the first Hund's rule determines the order of those $3 d^{n-1}$ multiplet states. For chargetransfer insulators on the other hand, the situation can be quite different. With $U$ larger than $\Delta$, the first ionization state will no longer be of the $3 d^{n-1}$ type but rather $3 d^{n} \underline{L}$, i.e., a state where the extra hole is in the O $2 p$ ligand rather than in the TM $3 d$ shell. Here we use the notation $L$ for the ligand hole. It turns out that such a first ionization state can have a spin character that is not given by the Hund's rule high-spin state. Instead, due to the strong anti-ferromagnetic interaction between the hole on the ligand and the hole on the TM ion, the state will be of spin-compensated nature. A famous example is the formation of the $d^{9} \underline{L}$ Zhang-Rice singlet instead of the $d^{8}$ triplet upon doping of a $\mathrm{Cu}^{2+}$ cuprate with holes $[10,11]$.

With $\mathrm{NiO}$ put in the intermediate regime of the ZSA phase diagram, it is not clear a priori whether the first ionization state has a high, or instead, a low spin character. This is not an unimportant issue since understanding of many of the intriguing properties of $\mathrm{Ni}^{3+}$ compounds like $\mathrm{RNiO}_{3}(\mathrm{R}=$ rare earth) and Li-doped $\mathrm{NiO}$ requires knowledge about the relative stability of the possible spin states. The question that needs to be addressed is whether the present day ab-initio theoretical calculations can take up the challenge to reliably determine these quantities. A review of the recent literature will be given below revealing that the results vary. To critically test the reliability or accuracy of the different theoretical approaches we will compare the calculated valence band spectrum with the one measured by photoelectron spectroscopy. Such a spectrum in fact represents one of the most fundamental excitations of the system, namely that one which is associated with the propagation of an extra particle injected into the system. Also here we will discuss the various theoretical approaches developed most recently and evaluate their strengths as well as possible shortcomings. 


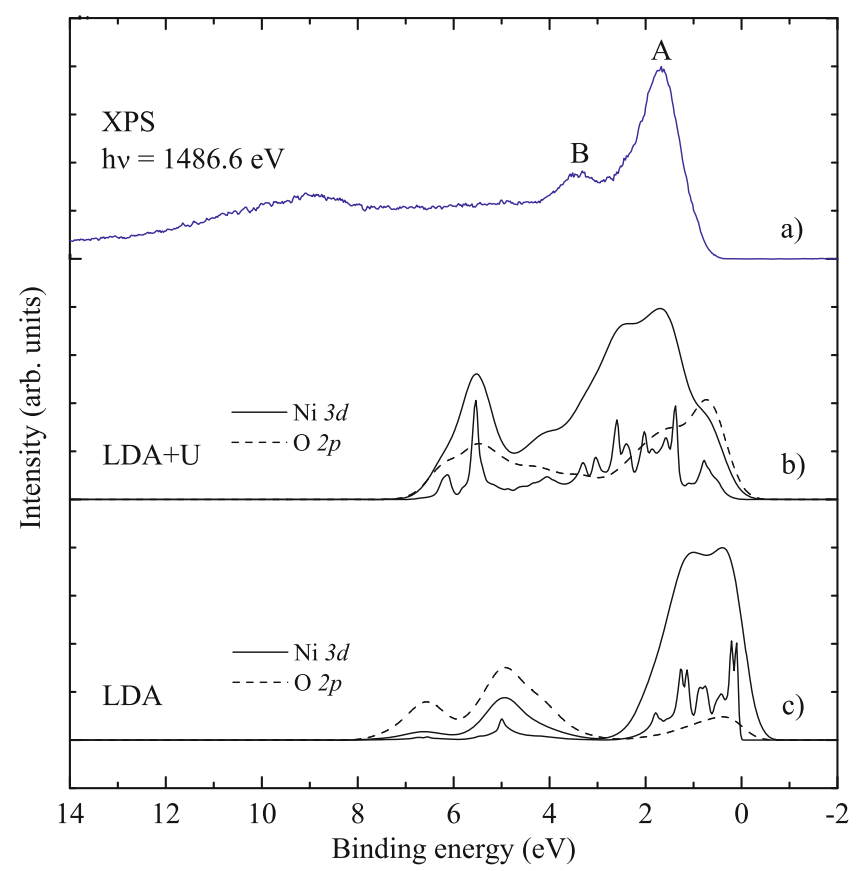

Fig. 1. Valence band (XPS) $(1486.6 \mathrm{eV})$ spectrum of an in situ cleaved $\mathrm{NiO}$ single crystal (blue curve) together with the results of LDA and LDA+U calculations (black curves), with and without the broadening to account for the experimental energy resolution.

\section{Valence band spectrum}

Figure 1 shows the valence band X-ray photoemission spectrum (XPS, $h \nu=$ $1486.6 \mathrm{eV}$ ) of a $\mathrm{NiO}$ single crystal cleaved in-situ. The measurement has been carried out at room temperature with an overall energy resolution of about $0.35 \mathrm{eV}$. The spectrum represents essentially the $\mathrm{Ni} 3 d$ spectral weight since the photo-ionization cross-sections of the $\mathrm{O} 2 p$ and the Ni $4 s$ orbitals are relatively small [12]. Figure 1 also shows the Ni $3 d$ density of states as calculated by band theory in the local density approximation (LDA). It is obvious that this deviates strongly from the experiment: the LDA spectrum has a Fermi cut-off and a completely different line shape than the experiment. The inclusion of correlation effects by using the LDA+U approach [13] does reproduce the insulating state but does not solve the line shape problem. All this confirms the shortcomings of static mean field theories to describe spectra associated with the fundamental one-particle Green's function of a strongly correlated system $[13,14]$.

Realizing that $\mathrm{NiO}$ is an insulator and that inter-Ni $3 d$ charge fluctuations are strongly suppressed due to strong Coulomb interactions, Fujimori and Minami [15] took a very different approach in which they ignored band dispersions all together and gave up the translational symmetry of the problem. Instead they used a local $\mathrm{NiO}_{6}$ cluster in which they implemented a configuration interaction model with full atomic multiplet theory. They were able to obtain an extremely good simulation of the experimental spectrum, see Figure 2. All characteristic spectral features seemed to be reproduced.

Nevertheless, this issue was revisited almost a decade later by van Elp et al. [16] motivated by the finding of Kuiper et al. [17] that upon Li-doping $\mathrm{NiO}$ has a large number of holes in the $\mathrm{O} 2 p$ band, strongly suggesting that $\mathrm{NiO}$ should be considered 


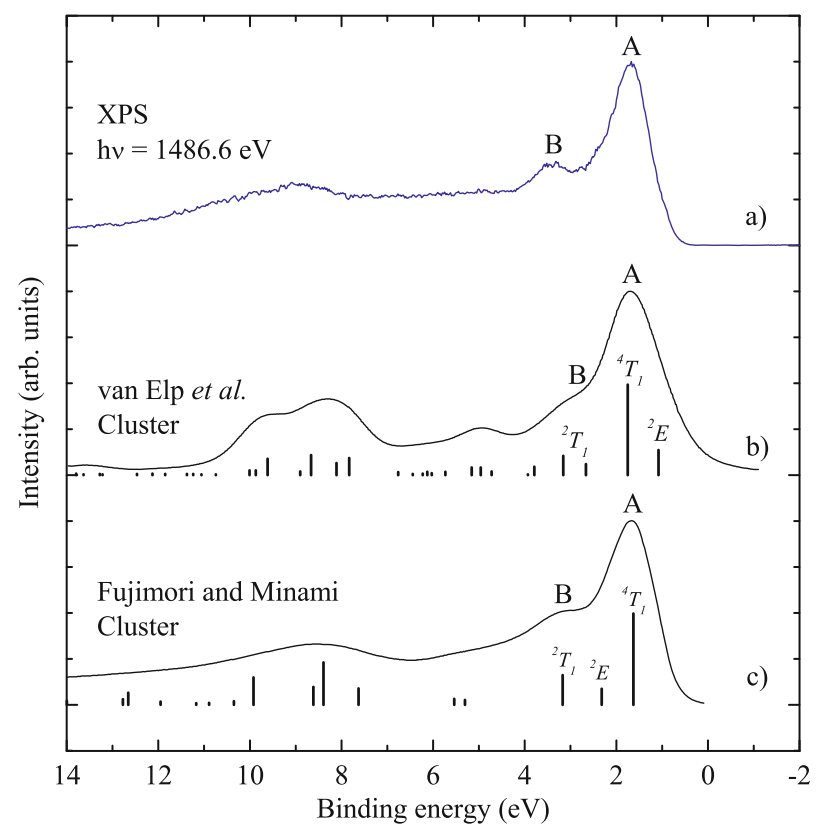

Fig. 2. Valence band XPS spectrum of $\mathrm{NiO}$ together with the results of single-site cluster configuration interaction calculations reproduced from references 15 and 16 , with and without the broadening to account for the experimental energy resolution.

in essence as a charge-transfer insulator. This is helpful to understand why the $\mathrm{Ni}^{3+}$ system $\mathrm{LiNiO}_{2}$ is low spin rather than high spin, since a charge-transfer insulator allows for low-spin solutions for the first ionization state of the parent compound $\mathrm{NiO}$. The cluster calculations by van Elp et al. [16] therefore were done with parameters such that the first ionization state is the so-called low spin ${ }^{2} E$ and not the high spin ${ }^{4} T_{1}$, i.e., opposite to the Fujimori and Minami solution, see Figure 2.

Interestingly, the spectral line shape of van Elp et al. has a less satisfactory match with the XPS experiment as compared to that of Fujimori and Minami. For example, the pronounced peak B in the experiment is much less present in the van Elp et al. spectrum, see Figure 2. In the following sections we will discuss in more detail how the spectral line shape and the character of the first ionization state depend on the magnitude of the Hubbard $U$ and the charge-transfer energy $\Delta$ and how inter-cluster effects influence the spectral line shape.

\section{Hubbard $U$ versus charge-transfer energy $\Delta$}

We have calculated the Ni $3 d$ spectral weight using a single Ni-site configurationinteraction cluster model which includes the full atomic multiplet theory as well as the Ni $3 d-\mathrm{O} 2 p$ ligand hybridization [18-20]. The simulations have been done for a $\mathrm{NiO}_{6}$ cluster in $O_{h}$ coordination using the program XTLS 8.3 [18] with parameter values which were determined from earlier studies on $\mathrm{NiO}[16,18,21,22]{ }^{1}$ We have varied the values of the Hubbard $U\left(U_{d d}\right)$ and the $\mathrm{O} 2 p$-Ni $3 d$ charge transfer energy,

${ }^{1}$ Parameters used for the calculation of the $\mathrm{NiO}_{6}$ cluster (in $\mathrm{eV}$ ): $\Delta=5.0$ if not specified otherwise, $U_{d d}=6.5$ if not specified otherwise, $10 D q=0.5, T_{p p}=0.8, V\left(e_{g}\right)=2.2, V\left(t_{2 g}\right)=$ $V\left(e_{g}\right) / 2, \zeta$ see reference [18], Slater integrals $70 \%$ of Hartree-Fock values. 


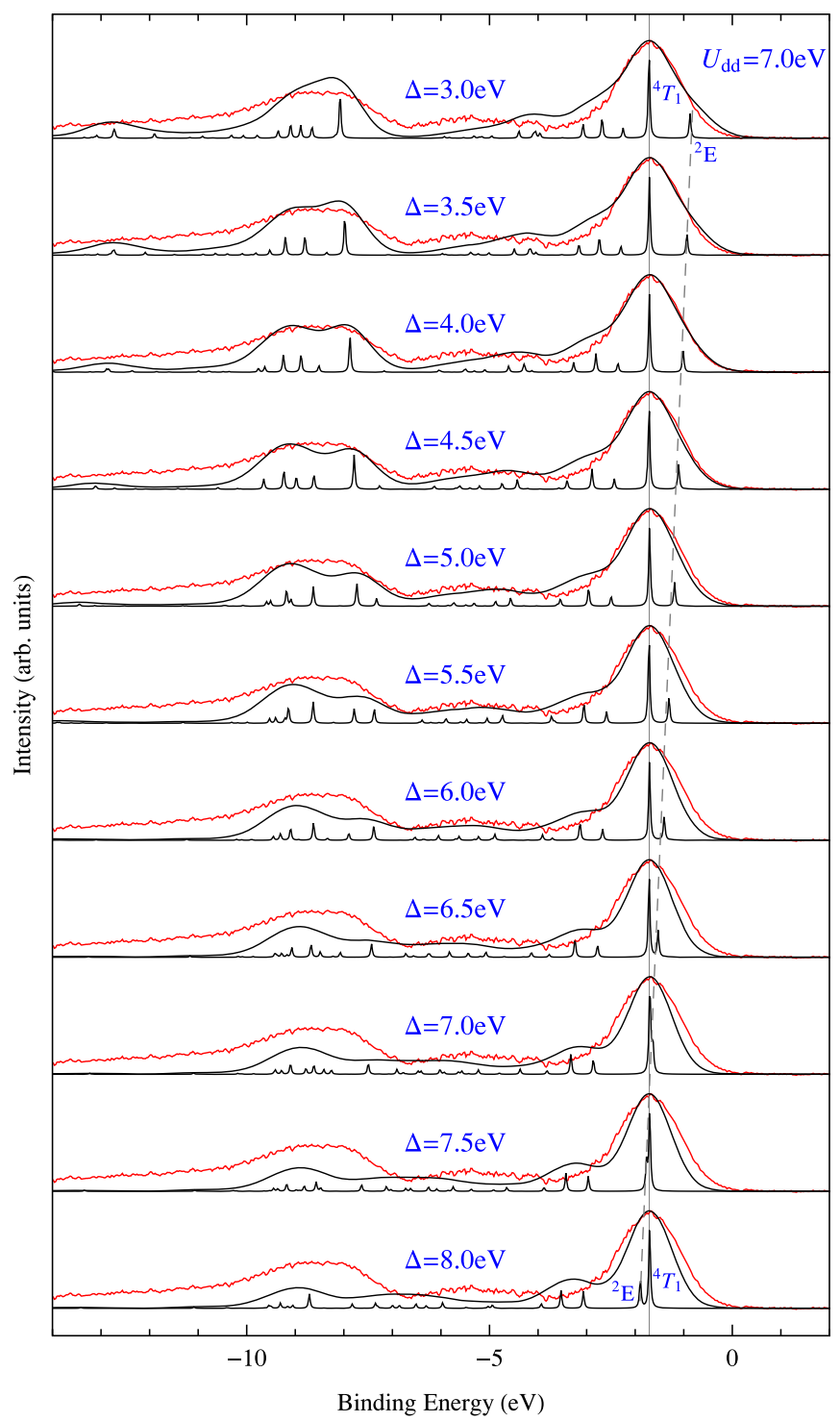

Fig. 3. Ni $3 d$ spectral weight from single-site $\mathrm{NiO}_{6}$ configuration-interaction cluster calculations for varying values of the charge-transfer energy $\Delta$ with the Hubbard $U\left(U_{d d}\right)$ set to $7.0 \mathrm{eV}$ (black curves), together with the experimental valence band (XPS) spectrum of a $\mathrm{NiO}$ impurity in $\mathrm{MgO}$ (red curves). The calculations have been carried out with $0.05 \mathrm{eV}$ FMWH (full width at half maximum) broadening (sharp peaks) and also with $1 \mathrm{eV} \mathrm{FWHM}$ broadening to account for the experimental energy resolution.

and a representative result is displayed in Figure 3 (black curves) for $U_{d d}=7.0 \mathrm{eV}$ with the $\Delta$ values varied between 3.0 to $8.0 \mathrm{eV}$. As a reference we have included in Figure 3 the Ni $3 d$ valence band photoemission spectrum of $\mathrm{Ni}_{0.05} \mathrm{Mg}_{0.95} \mathrm{O}$ [23], i.e., a $\mathrm{NiO}$ impurity system in $\mathrm{MgO}$ (red curves). We would like to note that it is more direct to compare the single-site calculations with a $\mathrm{NiO}$ impurity system than with the $\mathrm{NiO}$ bulk since the bulk may show inter-Ni-site or non-local screening effects that influences considerably the spectral line shape as we will show later. 


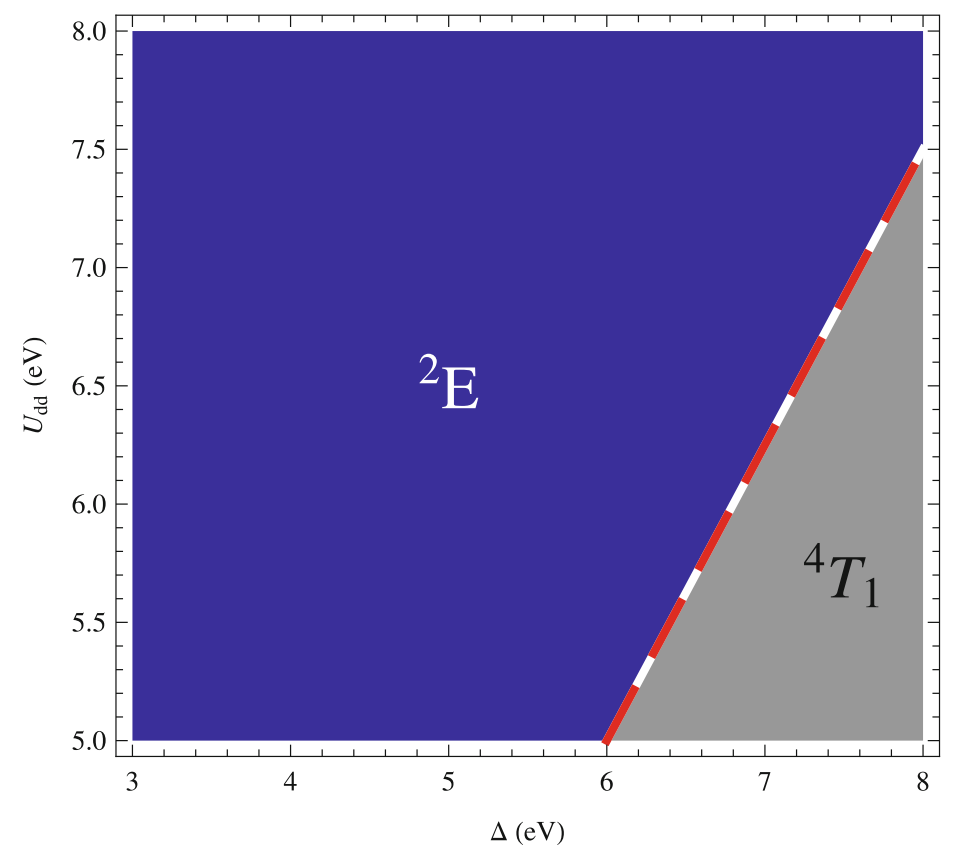

Fig. 4. Phase diagram of the first ionization state of $\mathrm{NiO}$ calculated using a $\mathrm{NiO}_{6}$ cluster: low spin ${ }^{2} E$ versus high spin ${ }^{4} T_{1}$.

Figure 3 reveals that a reasonable good match can be found between the simulations and the experiment for $\Delta$ values in the range of $4.0-5.0 \mathrm{eV}$. The lines shape of the main peak at $1.8 \mathrm{eV}$ binding energy (peak A) is well reproduced as well as the energy separation with the satellite structure at $7.5-10.0 \mathrm{eV}$ binding energies. For $\Delta$ values of $6.0 \mathrm{eV}$ or larger, we can observe appreciable discrepancies overall. In particular, it is worth noting that a new peak structure appear in the calculations at $3.4 \mathrm{eV}$ binding energy (peak B) which is not present in the experiment. From this we can learn that $U$ must be reasonably larger than $\Delta$ as to reproduce the single-site electronic properties of $\mathrm{NiO}$, i.e., that $\mathrm{NiO}$ is more on the charge-transfer side of the ZSA phase diagram rather than in the intermediate regime.

Another important result from Figure 3 is the observation that the first ionization state is given by the small intensity ${ }^{2} E$ peak for simulations with $\Delta$ values smaller than $U$. Reversely, if $U$ is smaller than $\Delta$ the first ionization state will be of the high intensity ${ }^{4} T_{1}$ character. From a more comprehensive set of $U$ and $\Delta$ variations we are able to create a crude phase diagram that shows under which conditions the low-spin or spin-compensated first ionization state is likely to be preferred above the high-spin one and vice-versa, see Figure 4. As already mentioned above, the first ionization state also represents the state that one will get if one dopes $\mathrm{NiO}$ chemically with holes. The phase diagram is thus also of relevance for the understanding of the low spin nature of $\mathrm{Ni}^{3+}$ systems like $\mathrm{LiNiO}_{2}$ and $\mathrm{RNiO}_{3}(\mathrm{R}=$ rare earth).

The difference between the Fujimori and Minami study [15] and that of van Elp et al. [16] is thus about what parameters to use as to simulate the experimental valence band spectrum best and what consequences the choice of parameters has for the spin character of the first ionization state. The Fujimori and Minami simulation had the better match to the experimental spectrum but ended up with the high spin scenario while van Elp et al. proposed the low spin picture at the expense of a poorer fit to the experimental spectrum. 


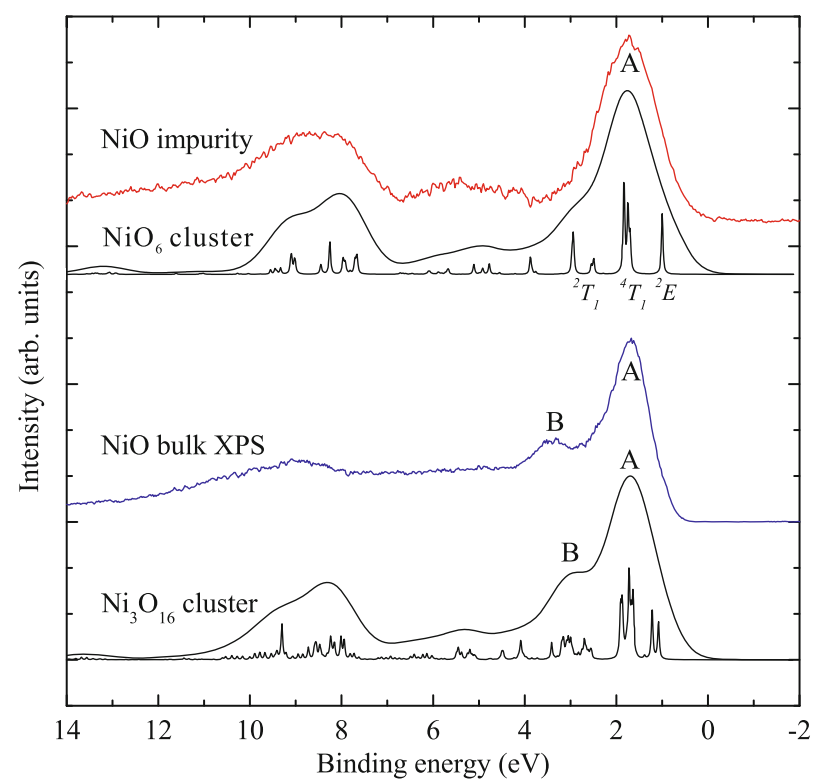

Fig. 5. Comparison of the valence band photoemission spectra of bulk $\mathrm{NiO}$ and $\mathrm{NiO}$ impurity in $\mathrm{MgO}$. Also included is the calculated $\mathrm{Ni} 3 d$ spectral weight from a single-Ni-site cluster $\left(\mathrm{NiO}_{6}\right)$ and a three-Ni-site cluster $\left(\mathrm{Ni}_{3} \mathrm{O}_{16}\right)$. The calculations have been broadened with $0.05 \mathrm{eV}$ FMWH (sharp peaks) and also with $1 \mathrm{eV} \mathrm{FWHM} \mathrm{to} \mathrm{account} \mathrm{for} \mathrm{the} \mathrm{experimental}$ energy resolution.

\section{Single-site versus multi-site effects}

The Ni $3 d$ valence band photoemission spectrum of $\mathrm{Ni}_{0.05} \mathrm{Mg}_{0.95} \mathrm{O}$ [23] used as reference in Figure 3 is actually quite remarkable. It differs in certain aspects essentially from that of bulk $\mathrm{NiO}$ shown in Figure 1, and if properly understood, one could arrive at a solution to the problem of how to have a good simulation of the experimental spectrum of bulk $\mathrm{NiO}$ and at the same time obtain a low spin first ionization state. We present the experimental data once again in Figure 5 for a detailed investigation. We can clearly observe that the main feature of the $\mathrm{NiO}$ impurity spectrum consists of a single peak $\mathrm{A}$ at $1.8 \mathrm{eV}$ binding energy, while that of bulk $\mathrm{NiO}$ has two peaks, namely peak $\mathrm{A}$ at $1.8 \mathrm{eV}$ and peak $\mathrm{B}$ at $3.4 \mathrm{eV}$.

To identify the origin of this striking difference, we present in Figure 5 a single-Nisite cluster calculation, namely a $\mathrm{NiO}_{6}$ octahedral cluster, that reproduces the $\mathrm{NiO}$ impurity spectrum rather well. The emphasis for this single-Ni-site simulation is to ensure that the calculated main peak does not show a pronounced peak B and that the first ionization state is the low-spin ${ }^{2} E$ state. The parameters used are listed in reference $^{1}$.

Next we carried out a multi-Ni-site cluster calculation in the form of a $\mathrm{Ni}_{3} \mathrm{O}_{16}$ chain made of three corner-shared $\mathrm{NiO}_{6}$ octahedra using the method developed by Tanaka [24] to reduce the many-body basis set. The spins of $\mathrm{Ni}$ ions in this short chain have antiferromagnetic correlations in the initial state. Important is that also in this calculation we are using the same values for all parameters as for the single Ni-site simulation. The calculated $\mathrm{Ni} 3 d$ one-electron removal spectrum on the central Ni ion is then displayed in Figure 5. We now can clearly observe that a new feature appears at $3.0 \mathrm{eV}$ binding energy, resembling very much peak $\mathrm{B}$ of the bulk $\mathrm{NiO}$ spectrum. We can expect that a $\mathrm{Ni}_{7} \mathrm{O}_{36}$ cluster in which a central $\mathrm{NiO}_{6}$ cluster is surrounded antiferromagnetically by six other $\mathrm{NiO}_{6}$ units, will produce an even more pronounced 
peak at 3.0-3.4 eV in Ni $3 d$ spectrum for the central Ni ion. This then basically should reproduce peak $\mathrm{B}$ of bulk $\mathrm{NiO}$.

We therefore can infer that the appearance of peak B must be sought in nonlocal correlations, i.e., effects which are beyond a single-site approach. An analogy may be drawn with the case of the $\mathrm{Ni} 2 p$ core level spectrum of $\mathrm{NiO}$. Also there a two-peak structure appears in the spectrum that is not present in a single Ni site cluster calculation. Van Veenendaal and Sawatzky [25] proposed a so-called non-local screening mechanism in which upon removal of a Ni $2 p$ core electron, an electron of a neighboring $\mathrm{NiO}_{6}$ cluster screens the localized $\mathrm{Ni} 2 p$ core hole thereby leaving behind a low-energy ${ }^{2} E$ state on the neighboring cluster.

To interpret the results of our multi-Ni-site cluster calculations in terms of the same non-local screening mechanism for our valence band case, we need to identify the existence of a high intensity peak in the valence band that is also rather localized in nature. Looking at Figure 5, we can see that the main peak is given by the ${ }^{4} T_{1}$ state. This state is formed by a hole that is injected by the photoemission experiment into the $t_{2 g}$ orbital starting from a high-spin $\mathrm{Ni} d^{8}$ ground state. The ability of this state to disperse is rather limited since the overlap between the $\mathrm{Ni}$ $3 d\left(t_{2 g}\right)$ and $\mathrm{O} 2 p(\pi)$ is small. We thus can consider this state as quasi-core. We then can invoke the non-local screening process as follows: After the creation of the ${ }^{4} T_{1}$ state, an $e_{g}$ electron from a neighboring $\mathrm{NiO}$ cluster hops onto the $\mathrm{Ni}$ site, leaving behind a coherent ${ }^{2} E$ hole state on that neighbor. These two states are energetically almost degenerate [25], and the Ni $3 d\left(e_{g}\right)$ and $\mathrm{O} 2 p(\sigma)$ hybridization between them is then strong enough to produce two peaks: Not only the main peak A but also peak B.

This non local screening mechanism is not applicable for the ${ }^{2} E$ state, which is a state in which a photohole is injected in an $e_{g}$ orbital starting from the $3 d^{8}{ }^{3} A_{2}$ ground state [22]. This hole can be expected to readily propagate in the lattice rather than being screened by the same type of hole on a neighboring site. Interestingly, although the transfer intergrals between the $\mathrm{Ni} 3 d\left(e_{g}\right)$ and $\mathrm{O} 2 p(\sigma)$ orbitals are rather large $[16,25]$, the effective nearest hopping of the ${ }^{2} E$ state can be expected to be very small due to the antiferromagnetic ordering. Indeed, the three Ni-site calculation show that the splitting in the ${ }^{2} E$ state is of order $0.2 \mathrm{eV}$ only.

\section{Antiferromagnetic order and electronic structure}

To quantify to what extent inter-Ni or non-local screening effects play a role in the valence band electronic structure of $\mathrm{NiO}$, we now investigate the influence of the antiferromagnetic order on the spectra. Figure 6 shows the valence band spectra taken at $300 \mathrm{~K}$ and at $525 \mathrm{~K}$, i.e., below and at the Neel temperature. We can clearly observe significant changes. The loss of antiferromagnetic order apparently causes a reduction of the height and an increase of the width of peak A. Also the energy separation between peak A and peak B becomes smaller.

These observations fits quite well with the non-local screening mechanism of the ${ }^{4} T_{1}$ main peak mentioned above. To show this we take a closer look at the electron removal states and the possible screening channels. Let us consider a spin-up Ni ion with the $d^{8}$ ground state, i.e., having two spin-down $e_{g}$ holes. The main peak in the photoemission spectrum of this ion is the high-spin ${ }^{4} T_{1}$ state, i.e., a state with the added hole in the spin-down $t_{2 g}$ orbital. In order to have the non-local screening from a neighboring $\mathrm{Ni}$ ion, i.e., to have the hopping of an $e_{g}$ electron from the neighbor leaving behind the ${ }^{2} E$ state on that neighbor, the $e_{g}$ electron must be spin-down, since the two $e_{g}$ holes available on the photoemitted Ni ion are both spin-down. This means that the neighboring $\mathrm{Ni}$ ion must be spin-down $d^{8}$ in the ground state. In other 


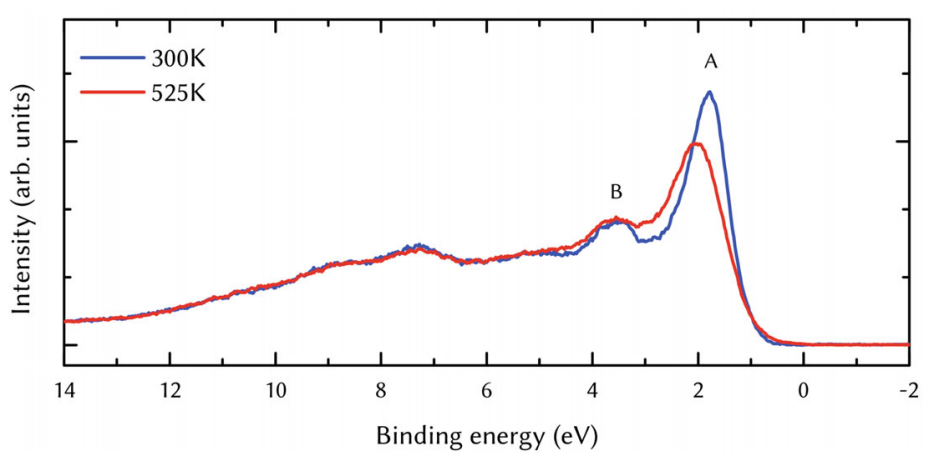

Fig. 6. $\mathrm{NiO}$ valence band spectra taken at 300 and $525 \mathrm{~K}$.

words, non-local screening is in this (simplified) picture only possible from nearest neighbors that are antiferromagnetically aligned. In the antiferromagnetic state, each $\mathrm{Ni}$ ion is surrounded by six antiferromagnetic neighbors. In the paramagnetic state, the number of antiferromagnetic neighbors is reduced to three on average. This means that non-local screening effects will be reduced in going from the antiferromagnetic to the paramagnetic state. The experimental spectral changes observed is consistent with these considerations, giving further support for our suggestion that multi Ni-site effects do play an important role in the electronic structure of $\mathrm{NiO}$.

\section{Recent theoretical developments}

A flurry of theoretical studies have been devoted recently to the $\mathrm{NiO}$ problem using a variety of new and highly sophisticated approaches. These include several types of GW calculations [26,27], LDA+DMFT [28-36], VCA (variational cluster approximation) [37-40] as well as calculations of the relevant screened interactions [41].

Focussing on the LDA+DMFT studies, we notice that they differ in the impurity solver utilized and in the implementation of the double counting correction. Nevertheless, it is quite encouraging that the calculations by Kunes et al. [29,30], Yin et al. [31], Miura et al. [32] and Nekrasov et al. [33] all produce quite similar Ni $3 d$ spectral weight: the first ionization state is of $e_{g}$ character and the main spectral feature consists of a single peak A. That the first ionization state is $e_{g}$ like is consistent with the experimental indication for the presence of the ${ }^{2} E$ state. However, the failure to reproduce peak $B$ visible in the experimental spectrum seems to indicate that peak $B$ indeed is a product of non-local screening effects which require an explanation beyond the single-site DMFT, i.e., a multi-site cluster DMFT is required. While the present DMFT results resemble in effect the single-site cluster findings of van Elp et al. [16], we would like to note that energy separation between the $e_{g}$ first ionization state and the $t_{2 g}$ main peak is much larger in the DMFT than in the van Elp study and in the experiment. Looking back at the set of simulations in Figure 3, one may infer that these DMFT studies somehow have overestimated considerably the Hubbard $U$ value relative to the charge-transfer energy $\Delta$.

Interestingly, the DMFT calculations by Thunström et al. [34] as well as the VCA by Eder [38-40] do produce a two peak structure for the main feature, although one might object that the relative weights of the two peaks are quite different from the experimental peak A to peak B ratio. Most important is that the first ionization state is of $t_{2 g}$ character (and thus ${ }^{4} T_{1}$ like) in these calculations, in disagreement with the low spin nature of $\mathrm{Ni}^{3+}$ oxides. In fact, one could infer that these results 


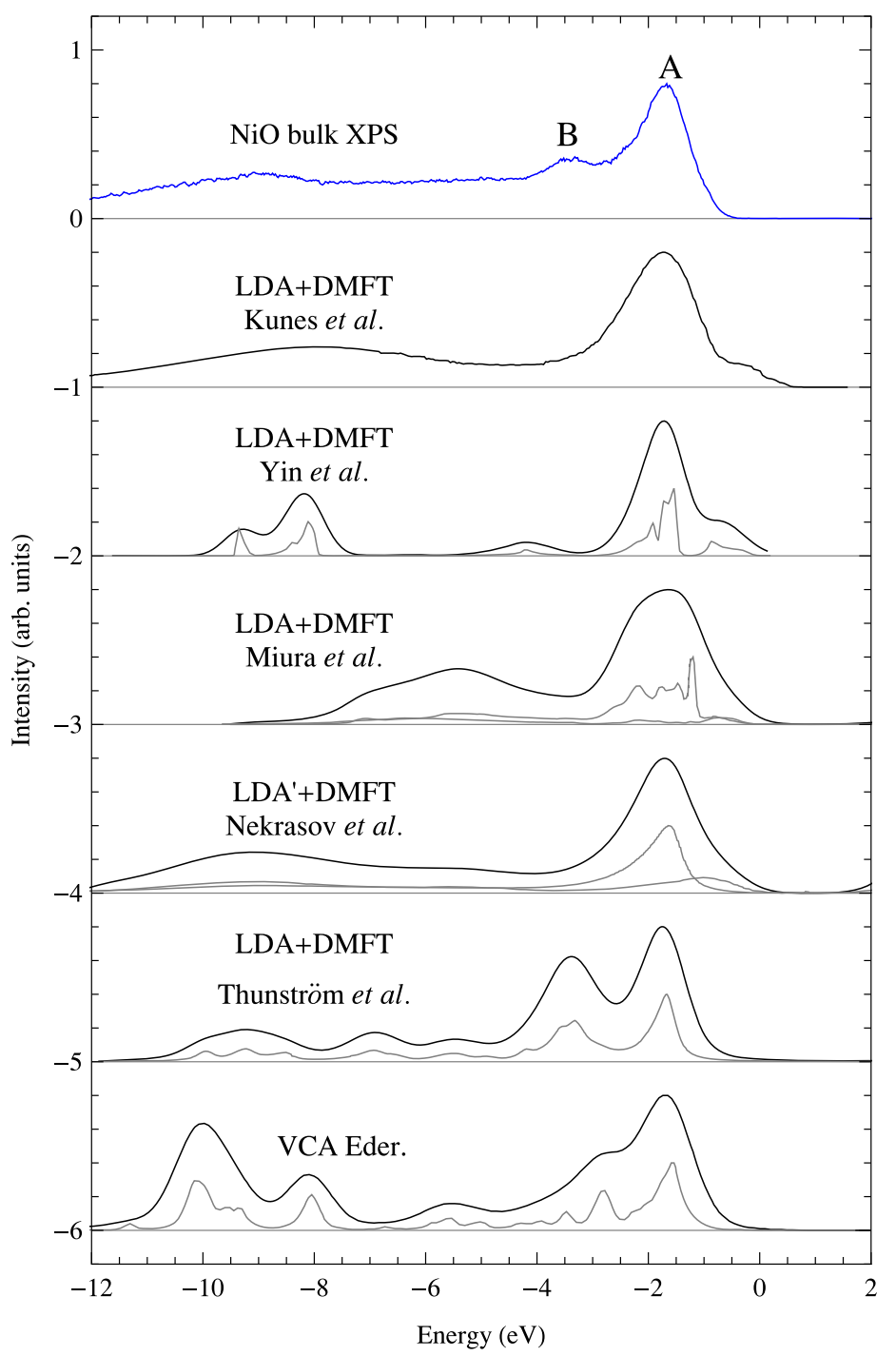

Fig. 7. Valence band XPS spectrum of $\mathrm{NiO}$ (blue curve) together with the Ni $3 d$ spectral weight from recent LDA + DMFT and VCA calculations (black lines with $1 \mathrm{eV}$ FWHM broadening, grey dashed lines as originally given in the references).

are quite similar to the early single-site cluster calculations by Fujimori and Minami [15] and that the Hubbard $U$ value has probably been underestimated relative to the charge-transfer energy $\Delta$. All this shows the importance of developing methods to determine reliable values for the screened interactions [41] and to correct for possible double counting of the interactions $[33,42]$.

\section{Conclusions}

We have investigated the electronic structure of $\mathrm{NiO}$ using a combination of photoelectron spectroscopy and cluster configuration interaction calculations. We have 
found that $\mathrm{NiO}$ is a charge-transfer insulator with a spin-compensated first ionization state, thereby explaining the tendency of $\mathrm{Ni}^{3+}$ oxides to form low-spin systems. From studying experimentally the temperature and dilution dependence as well as from analyzing the cluster size dependence of the spectral line shape we have also established the importance of non-local screening effects. These findings call for a consistent ab-initio determination of the charge transfer energy $\Delta$ and the Hubbard $U$ and point out the need to include multi-site interactions in the quantitative ab-initio modeling of the electronic structure of transition metal oxides.

We gratefully acknowledge support of the Deutsche Forschungsgemeinschaft through FOR 1346. Open access funding provided by Max Planck Society.

Open Access This is an Open Access article distributed under the terms of the Creative Commons Attribution License (http://creativecommons.org/licenses/by/4.0), which permits unrestricted use, distribution, and reproduction in any medium, provided the original work is properly cited.

\section{References}

1. W.L. Roth, Phys. Rev. 110, 1333 (1958)

2. J.H. de Boer, E.J.W. Verwey, Proc. Phys. Soc. 49, 59 (1937)

3. N.F. Mott, Proc. Phys. Soc. London A 62, 416 (1949)

4. J. Hubbard, Proc. R. Soc. London A 276, 238 (1963)

5. T. Oguchi, K. Terakura, A.R. Williams, Phys. Rev. B 28, 6443 (1983)

6. K. Terakura, A.R. Williams, T. Oguchi, J. Kübler, Phys. Rev. Lett. 52, 1830 (1984)

7. K. Terakura, T. Oguchi, A.R. Williams, J. Kübler, Phys. Rev. B 30, 4734 (1984)

8. G.A. Sawatzky, J.W. Allen, Phys. Rev. Lett. 53, 2339 (1984)

9. J. Zaanen, G.A. Sawatzky, J.W. Allen, Phys. Rev. Lett. 55, 418 (1985)

10. F.C. Zhang, T.M. Rice, Phys. Rev. B 37, 3759 (1988)

11. H. Eskes, G.A. Sawatzky, Phys. Rev. Lett. 61, 1415 (1988)

12. J.J. Yeh, I. Lindau, At. Data Nucl. Data Tables 32, 1 (1985)

13. V.I. Anisimov, J. Zaanen, O.K. Andersen, Phys. Rev. B 44, 943 (1991)

14. V.I. Anisimov, I.V. Solovyev, M.A. Korotin, M.T. Czyzyk, G.A. Sawatzky, Phys. Rev. B 48, 16929 (1993)

15. A. Fujimori, F. Minami, Phys. Rev. B 30, 957 (1984)

16. J. van Elp, H. Eskes, P. Kuiper, G.A. Sawatzky, Phys. Rev. B 45, 1612 (1992)

17. P. Kuiper, G. Kruizinga, J. Ghijsen, G.A. Sawatzky, H. Verweij, Phys. Rev. Lett. 62, $221(1989)$

18. A. Tanaka, T. Jo, J. Phys. Soc. Jpn. 63, 2788 (1994)

19. F.M.F. de Groot, J. Electron. Spectrosc. Relat. Phenom. 67, 529 (1994)

20. See the Theo Thole Memorial Issue, J. Electron, Spectrosc. Relat. Phenom. 86, 1 (1997)

21. D. Alders, L.H. Tjeng, F.C. Voogt, T. Hibma, G.A. Sawatzky, C.T. Chen, J. Vogel, M. Sacchi, S. Iacobucci, Phys. Rev. B 57, 11623 (1998)

22. M.W. Haverkort, S.I. Csiszar, Z. Hu, S. Altieri, A. Tanaka, H.H. Hsieh, H.-J. Lin, C.T. Chen, T. Hibma, L.H. Tjeng, Phys. Rev. B 69, 020408 (2004)

23. T. Haupricht, PhD thesis, University of Cologne, 2011

24. A. Tanaka, J. Phys. Soc. Jpn. 68, 3493 (1999)

25. M.A. van Veenendaal, G.A. Sawatzky, Phys. Rev. Lett. 70, 2459 (1993)

26. H. Jiang, R.I. Gomez-Abal, P. Rinke, M. Scheffler, Phys. Rev. B 82, 045108 (2010)

27. S. Das, J.E. Coulter, E. Manousakis, Phys. Rev. B 91, 115105 (2015)

28. X. Ren, I. Leonov, G. Keller, M. Kollar, I. Nekrasov, D. Vollhardt, Phys. Rev. B 74 $195114(2006)$

29. J. Kunes, V.I. Anisimov, A.V. Lukoyanov, D. Vollhardt, Phys. Rev. B 75, 165115 (2007) 
30. J. Kunes, V.I. Anisimov, S.L. Skornyakov, A.V. Lukoyanov, D. Vollhardt, Phys. Rev. Lett. 99, 156404 (2007)

31. Q. Yin, A. Gordienko, X. Wan, S.Y. Savrasov, Phys. Rev. Lett. 100, 066406 (2008)

32. O. Miura, T. Fujiwara, Phys. Rev. B 77, 195124 (2008)

33. I. Nekrasov, V. Pavlov, M. Sadovskii, JETP Lett. 95, 581 (2012)

34. P. Thunström, I. Di Marco, O. Eriksson, Phys. Rev. Lett. 109, 186401 (2012)

35. Z. Jian-Zhou, Z. Jia-Ning, D. Xiao-Yu, B. Yan, C. Ling-Cang, F. Zhong, D. Xi, Chin. Phys. B 21, 057106 (2012)

36. A. Hariki, Y. Ichinozuka, T. Uozumi, J. Phys. Soc. Jpn. 82, 043710 (2013)

37. R. Eder, A. Dorneich, H. Winter, Phys. Rev. B 71, 045105 (2005)

38. R. Eder, Phys. Rev. B 76, 241103 (2007)

39. R. Eder, Phys. Rev. B 78, 115111 (2008)

40. R. Eder, Phys. Rev. B 91, 245146 (2015)

41. R. Sakuma, F. Aryasetiawan, Phys. Rev. B 87, 165118 (2013)

42. M. Karolak, G. Ulm, T. Wehling, V. Mazurenko, A. Poteryaev, A. Lichtenstein, J. Elec. Spectosc. Rel. Phen. 181, 11 (2010) 